\section{RMD Open}

Rheumatic \&

Musculoskeletal Diseases

\title{
Update on interleukin-17: a role in the pathogenesis of inflammatory arthritis and implication for clinical practice
}

\section{Pierre Miossec}

To cite: Miossec P. Update on interleukin-17: a role in the pathogenesis of inflammatory arthritis and implication for clinical practice. RMD Open 2017;3: e000284. doi:10.1136/ rmdopen-2016-000284

- Prepublication history and additional material for this paper is available online. To view these files please visit the journal online (http://dx.doi.org/10.1136/ rmdopen-2016-000284).

Received 7 December 2016 Revised 16 January 2017 Accepted 17 January 2017

Immunogenomics and Inflammation Research Unit EA 4130, Department of Immunology and Rheumatology, University of Lyon, Lyon, France

Correspondence to Professor Pierre Miossec; pierre.miossec@univ-lyon1.fr

\section{ABSTRACT}

Interleukin-17 (IL-17A) is a cytokine critical for the acute defence against extracellular bacterial and fungal infections. Excess production during chronic inflammation has been associated with many inflammatory and autoimmune disorders. The present review describes the key molecules of the IL-17 pathway, which are or could be targeted for treatment. Since targeting of IL-17A may affect defence mechanisms, the pathogenesis of such possible adverse events is analysed. Then the contributions of IL-17 to bone changes in various forms of arthritis are discussed. Finally, the results of current inhibitors of the IL-17 pathway in clinical trials are detailed. IL-17A inhibition has been first registered for the treatment of psoriasis, psoriatic arthritis and ankylosing spondylitis. Other therapeutic options are now tested in a long list of diseases.

Interleukin-17 (IL-17, now IL-17A) is now a recognised target in chronic inflammation. ${ }^{1}$ This cytokine was described in 1993-1995 and its first inhibitor was registered in 2015. Previous and ongoing research has identified a long list of clinical conditions where IL-17A and related molecules are important contributors. In parallel, the number of options to target these cytokines, their receptors, transcription factors and signalling pathways is growing quickly.

The basis for such development has started as early as two decades ago with in vitro results identifying its contribution to inflammation. ${ }^{2}$ At the same time, novelty resulted from the discovery that both IL-17A and its receptor were part of a novel family of ligands and cytokine receptors. Identification of patients with defects of the IL-17 pathway provided reasons to address properly the risk of adverse events during IL-17 targeting.

Over the years, a long list of publications has associated IL-17A with many functions in cell biology and its association as well as contribution to an increasing number of diseases. ${ }^{3}$ With clinical applications in mind, this review will focus on some of these

\section{Key messages}

What is already known about this subject?

- Previous studies have indicated the role of II-17 in chronic inflammation. This is the basis for the use of IL-17 inhibitors in arthritis.

What does this study add?

- The use of IL-17 inhibition in psoriatic arthritis (PsA) is easy to understand because of the role of IL-17 in bone destruction. However, the use of the same inhibitors for the treatment of ankylosing spondylitis (AS) implies another understanding. In fact, IL-17 specifically in combination with TNF, induces bone matrix formation by isolated osteoblasts. This is the case at the site of ligaments insertion, where osteoclasts are absent. Local bone formation leads to syndesmophytes.

How might this impact on clinical practice?

- This new understanding justifies how inhibition of IL-17 may be active in a bone destructive disease, such as PsA, as well as in a disease associated in some location with ectopic bone formation, as in AS.

aspects with direct clinical relevance. To do so, we will focus on the function of the key molecules of the IL-17 pathway, which are or could be drug targets. Then we will analyse the contribution of IL-17A to host defence to understand and prevent the risk of specific infections during its targeting. We will also explain the heterogeneity of the effects of IL-17A on bone matrix destruction and formation to justify its inhibition in arthritis diseases with opposite features affecting the bone. Finally, we will conclude on its targeting today and tomorrow.

Key molecules from the IL-17 pathway as current and future drug targets

IL-17A was first described in 1993 as the product of the CTLA8 gene identified in the mouse and human genomes. ${ }^{4}$ Its association 
with $\mathrm{T}$ cells provided a new understanding of the role of $\mathrm{T}$ cells in some diseases such as rheumatoid arthritis (RA), at a time when such contribution was still a debate. ${ }^{5}$ Although the start of the IL-17A discovery was difficult since no effects on $\mathrm{B}$ and $\mathrm{T}$ cells were seen, the first results linked IL-17A to inflammation by the demonstration of the induction of IL-6 production by synoviocytes. ${ }^{26}$ At the same time, its effect on neutrophil differentiation by inducing granulocyte colony-stimulating factor and on neutrophil migration by inducing IL-8 established a firm connection between IL-17A and neutrophil biology and associated diseases. ${ }^{2}$ This will be further discussed later.

Based on sequence analysis of genes and proteins, the family of IL-17A was next described and now includes six members. ${ }^{7}$ The first IL-17 was renamed IL-17A. Among the other family members, IL-17F and IL-17E are the most important. IL-17F is the closest member to IL-17A and shows a $50 \%$ sequence identity with IL-17A. IL-17F alone is less active than IL-17A, but appears to be biologically important. In a context of inflammation driven by cytokines such as tumour necrosis factor (TNF), there is a clear synergy between TNF and IL-17F, reaching a level of proinflammatory gene signature not very far from that induced by the combination of TNF and IL-17A. ${ }^{8}$

Opposite to IL-17A and IL-17F, IL-17E, more commonly referred to as IL-25, shows the lowest homology with IL-17A of only $19 \%$. Whereas both IL-17A and IL-17F induce an inflammatory response, IL-25 can downregulate such effect. ${ }^{10}$ This regulation is of interest to be understood. IL-17A, specifically when combined with other proinflammatory cytokines, induces a rapid inflammatory response, as detected for instance by the production of IL-6 or IL-8. At a late stage, the production of IL-25 starts, leading to an endogenous control of the inflammatory response. When such control is in place, it will lead to the cessation of the acute IL-17A-driven response. Conversely, a defect in the IL-17A/IL-25 balance in favour of IL-17A will lead to a chronic situation where the amount of IL-25 is not large enough to control the effect of IL-17A. Similar regulation was described many years ago for IL-1 and its receptor antagonist IL-1RA.

The receptor of IL-17A was identified in 1995 at the same time IL-17A was discovered. ${ }^{11}{ }^{12}$ It was first classified as the first member of a new cytokine receptor family. As for the protein family, comparison of protein sequences identified the IL-17 receptor family. The first described IL-17R was renamed IL-17RA. The full functional receptor combines the IL-17RA chain to the IL-17RC chain. This receptor binds IL-17A and IL-17F. ${ }^{13}$ Thus, inhibition of the first IL-17R or IL-17RA will inhibit the effects of IL-17A and IL-17F.

The receptor of IL-25, the natural inhibitor of IL-17A and IL-17F, uses the same IL-17RA chain, which can thus be defined as a common chain, and an IL-25 specific chain, IL-17RB. In conclusion, these results now show that the specific inhibition of the IL-17RA chain will have the expected anti-inflammatory effects by blocking IL-17A and IL-17F. At the same time, such inhibition could also inhibit the binding of IL-25, thus possibly reducing its anti-inflammatory effects. This may suggest a clinical difference in efficacy and adverse events between the anti-IL-17A antibodies and the anti-IL-17RA antibody.

IL-17A and IL-17F are produced by a large variety of lymphocytes including the typical $\mathrm{T}$ helper 17 (Th17) cells as well as the $\gamma \delta \mathrm{T}$ cells, natural killer (NK) cells and nNKT cells. ${ }^{14}$ This long list of producing cells recognises the contribution of the IL-17 pathway to the innate and acquired immunity. The differentiation of a naive $\mathrm{T}$ cell or lymphocyte involves the expression of the lineagespecific transcription factor Roryt in the mouse, or Rorc in the human system. ${ }^{15}$ Inhibition of Roryt inhibits Th17 differentiation and the production of IL-17A and IL-17F, as well as of the other Th17 cytokines IL-21 and IL-22. Such an intracellular transcription factor can now be targeted with small molecules.

Upstream, the differentiation of the Th17 and other IL-17-producing cells is under the control of a series of steps that involve different cytokines produced by antigen presenting cells such as dendritic cells. Some of these cytokines have rather extended and non-specific inflammatory effects on multiple targets. IL-6 and IL-1, classical markers of inflammation, are critical cytokines for the differentiation of Th17 cells. Following early differentiation and expression of Ror $\mathrm{t}$, the final step is under the control of IL-23. IL-23 is a member of the IL-12 family as it shares with IL-12 the p40 common chain combined either with p19 to form IL-23, or p35 to form IL-12. ${ }^{16}$ Thus, the specific inhibition of IL-23 cannot be achieved by blocking p40, which blocks both IL-12 and IL-23. Instead, a specific inhibitor of p19 is needed to reach that specificity. Inhibition of p19IL-23 affects the differentiation of $\mathrm{T}$ cells into Th17 cells, thus reducing their cytokine production. This includes clearly that of IL-17A and IL-17F and also that of the other Th17 cytokines IL-21 and IL-22. Similar effects but not identical results are seen with the inhibition of Roryt.

In parallel, there is a balance between effector Th17 cells and regulatory $\mathrm{T}$ cells. ${ }^{17}$ In patients with RA, the loss of response to TNF inhibition appears to result from a change in such balance in favour of the Th17 side. $^{18}$

\section{The IL-17A pathway and the control of infections}

Safety comes first when targeting a pathway involved in the control of infections. $T$ cells are critical for the control of infections and the related primary immune deficiencies are usually much more severe with patients very sensitive to infections. The definition of subsets of $\mathrm{T}$ cells has been associated with subsets of infectious agents that they control. Patients with defects in the interferon $\gamma$ (IFNg) protein and receptor complex develop intracellular bacterial infections, including severe lethal diffuse infection when vaccinated with the 
attenuated but live BCG. This defines the protection affected to the Th1 pathway. At a lower level, patients with chronic arthritis also have a cell mediated immune defect that affects specifically the $\beta$ chain of the IL-12 receptor, leading to a reduction in the production of IFN $\gamma$. This contributes to the risk of reactivation of tuberculosis when blocking TNF. ${ }^{19}$ Conversely, parasitic infections are under the control of the Th2 pathway characterised by the production of IL-4 and IL-13, with anti-inflammatory properties.

For many years until 2005, when the concept of the Th17 pathway was discovered, no link was established between a T-cell subset and extracellular bacterial infections. These very common infections are associated with high neutrophil counts, and normal neutrophil functions are critical for the control of these infections. Regarding the primary deficiencies, patients with defects in the Th17 pathway develop severe staphylococcus and candida infections. Several defects have been isolated such as the hyper IgE syndrome with a defect in the Stat3 transcription factor and the chronic mucocutaneous candidiasis with either a reduced production of IL-17A or IL-17F or their inhibition with anti-IL-17A autoantibodies. ${ }^{20}$ Based on these observations, bacterial and candida infections are to be expected when blocking the IL-17 pathway. However, their severity should be reduced compared with that seen in patients with a genetic defect. Conversely, the risk of tuberculosis should not be affected. ${ }^{21}$

\section{Effects of IL-17A on local and systemic inflammation}

IL-17A and, to a lower extent, IL-17F induce an inflammatory signature in all mesenchymal cells with rather minor differences related to the anatomical origin of the cell. A high proportion of this signature is linked to neutrophil formation, migration and activation. In addition, activation of the chemokine CCL20 favours the migration of immature dendritic cells and Th17 cells. ${ }^{22}$ This effect induces and perpetuates the formation of an immune reaction at an ectopic lymphoid site. Interaction between these migrating Th17 cells and the local mesenchymal cells leads to a massive secretion of IL-17 at this site. ${ }^{23}$

Although predominantly acting at the local site, IL-17A can circulate in blood and thus may affect endothelial cells. On these cells, IL-17A, specifically in combination with TNF, induces a procoagulant state, which, combined with an increased platelet aggregation, induces local thrombosis. ${ }^{24}$ This could explain the IL-17 peak in the blood of patients with acute myocardial infarction. ${ }^{25}$ The effect of IL-17A on muscle cells may further contribute to cardiac dysfunction. ${ }^{26}$

\section{Heterogeneous effects of IL-17A on bone}

In the context of arthritis, the concept of targeting inflammation and its cytokines is based on their contribution to bone metabolism. The simplest understanding is the destructive properties of cytokines on bone.
Transferred to the clinical situation, this explains the destruction of bone in inflammatory destructive arthritic such as RA, juvenile idiopathic arthritis psoriatic arthritis (PsA). Similar understanding applies when inflammation is induced by infections, or exogenous particles as in parotonditis, septic and aseptic joint prosthesis loosening. Although it might be easier to understand destruction because it is easily seen, this clinical destruction results in fact from a massive inhibition of repair. This has led to the use of TNF inhibitors to control destruction. Indeed, control of destruction can be observed in the clinic but the induction of a repair activity is still missing.

Using samples of bone obtained from surgery, addition of IL-17A, even more when combined with TNF and IL-1, induced in vitro bone-destructive cytokine production and bone resorption. ${ }^{27}$ Conversely, inhibition of IL-17A resulted in an anti-inflammatory effect and lower bone destruction. In addition, a better effect was obtained when combining inhibitors of IL-17A, TNF and IL-1 since some samples appear to be driven more by one out of the three cytokines. ${ }^{28}$ These results are in line with those in animal models and human trials.

The situation of bone in ankylosing spondylitis (AS) is more complex. Clearly the same systemic bone loss is obvious in these patients with the extreme frailty of vertebral bone. In sharp contrast is the situation in the syndesmophytes in AS. There the opposite is seen with ectopic new bone formation. In that case, excessive bone formation is the issue, resulting in the clinical spinal stiffness. In this context of AS, if, as discussed above, cytokines induce bone destruction, how can we justify the use of TNF inhibitors if inhibition of destruction and even induction of repair is the consequence of such treatment? Either there should be a contraindication or short-term use, unless there is another explanation. Inhibition of TNF has been registered for AS and PsA and this has been extended to IL-17A inhibition.

A simple understanding would imply that IL-17A and the other cytokines would then inhibit osteoblast differentiation and function. This is indeed seen when looking at whole AS bone..$^{29}$ However, the effect is opposite when mesenchymal cells from different origin are incubated with the bone differentiation medium in the presence of IL-17A, alone and combined with TNF. Surprisingly, this leads to the formation of extracellular calcified bone matrix with an increase in alkaline phosphatase activation. ${ }^{31} \mathrm{TNF}$ alone has such an effect, but this effect is synergistically increased when TNF and IL-17A are combined.

Looking at the various genes involved in osteoblast differentiation, the Schnurri3 gene was found to be of interest. This gene has been recently studied since deficient mice show an increased bone mass. ${ }^{32}$ Schurri3 function induces a bridge through which osteoblasts and osteoclasts communicate and its expression is synergistically increased when TNF and IL-17A are combined. ${ }^{33}$ When osteoblasts and osteoclasts interact physically as in whole bone, this results in an activation of osteoclasts leading to 
bone resorption and lack of repair. However, when such a bridge is not present, the very same cytokines will now induce new bone formation. ${ }^{31}$ In normal bone repair, this effect is seen in the periosteum to induce, for instance, fracture repair. In an abnormal situation, such as in the syndesmophytes when mesenchymal cells from vertebral ligaments are inflamed in the physical absence of osteoclasts, this will induce new bone formation.

If these observations are translated to the clinic, inhibition of IL-17A should reduce bone destruction in PsA and systemic AS bone. In contrast, the opposite effect should be seen in syndesmophyte formation. It is too early to predict if this can still be seen once the disease is fully established. This implies that the best results will be seen if such treatment is given early.

\section{Current applications of IL-17 pathway targeting}

The concepts and therapeutic options which are already in place are based on discoveries and patents from 1995 to 1996 . This includes only the targeting of IL-17, now IL-A, and of IL-17R, now IL-17RA. ${ }^{34}$

Secukinumab has been approved for the treatment of psoriasis by the European Medicines Agency (EMA) and the Food and Drug Administration (FDA) in early 2015, based on very impressive results on skin lesions. ${ }^{35}$ These effects are described by the dermatologists as never seen before. Indeed, and for the first time, the 90 even 100\% disappearance of the skin lesions could be achieved. In early 2016, Ixekizumab got the same approval with the same impressive results in psoriasis. ${ }^{36}$ Finally, brodalumab in the process of getting approval got similar clinical results through the targeting of the IL17RA chain of the IL-17 receptor. ${ }^{37}$

Secukinumab has later been approved for the treatment of PsA and AS at the end of 2015 by the EMA and the beginning of 2016 by the FDA. ${ }^{38}$ Ixekizumab will be applying for approval for the same indications. ${ }^{39}$ Although these new drugs add to the choice that for a long time was limited to TNF inhibitors, the results on arthritis are not as impressive as on the skin. One simple explanation is that the skin lesions are just related to inflammation and cell proliferation, without destruction. The situation is more complex when bone matrix changes are affected. It is still difficult to expect an induction of a repair activity of a destroyed joint as in PsA. Even more difficult is to expect an effect on already established syndesmophytes. New trials will be needed in patients with early disease to prove that blocking IL-17A can prevent destruction in PsA and ectopic bone formation in AS. Based on the preclinical understanding discussed above, it is plausible to expect an effect with a single drug acting on two opposite targets.

Adverse events for the three drugs were as expected, including bacterial infections and localised candida infections, which were easily controlled. With brodalumab but not with the two antibodies, suicides and suicidal ideations were observed, leading to a caution in the labelling of the marketing authorisation. ${ }^{40}$ No explanation has been provided for this adverse event. It is still unclear whether the difference between the two modes of inhibition is real. The specificity of the inhibition of the IL-17RA versus IL-17A suggests a differential ligand receptor interaction. One option suggests the contribution of IL-25 with anti-inflammatory effects, which is inhibited with an anti-IL-17RA antibody. This could be tested in samples from these patients, but unfortunately, access to those appears difficult.

\section{Future applications of IL-17 pathway targeting}

Based on the description of the key molecules of the IL-17 pathway, many tools are now tested in the same and different conditions.

Owing to the structural similarities between IL-17A and IL-17F, the targeting of both is tested with antibodies targeting common epitopes on IL-17A and IL-17F. Increased efficacy can be expected. It is unclear if the overall good safety profile of IL-17A inhibition will be modified. ${ }^{34}{ }^{41}$ Targeting of IL-17A and IL-17F with a bispecific antibody is based on the contribution of both cytokines to increase the proinflammatory effects of TNF and similar cytokines, often in a synergistic manner. Although the effect of IL-17F is lower than that of IL-17A, the number of IL-17F-producing cells and the resulting local and systemic levels are higher. ${ }^{8}$ In addition, blocking IL-17A and IL-17F appears more potent than blocking IL-17A alone to reduce the inflammatory properties of supernatants of Th17 clones. $^{42}$ This could provide a better effect in the clinic to control more fully the inflammation driven by bioactive IL-17A and IL-17F. Furthermore, this could reduce the heterogeneity of response seen with to inhibition of IL-17A alone. ${ }^{43}$ The lack of such response may result from the persistence of IL-17F contribution and/or that of high levels of IL-25.

Bispecific antibodies targeting both IL-17A and TNF are now tested in trials. Based on the synergistic interactions described above, increased efficacy could be expected. ${ }^{34}$ At the same time, the situation is more complex than was first thought. Specifically, the synergistic interaction between TNF and IL-17A is seen if and only if, IL-17A acts first, then followed by TNF but not the other way around. It is unclear how a bispecific antibody recognising with one arm TNF and with the other IL-17A would act in vivo at the disease site. The next question is the advantages and limits of using two inhibitors versus a single bispecific molecule.

As with the use of small molecules targeting the Jak/Stat pathway versus biologics, the Roryt/Rorc transcription factor is the target of small molecules developed by many companies. It is too early to tell how they will compare with the inhibition with biologics of IL-17 molecules.

The list of diseases where IL-17A could be targeted is growing quickly. This includes diseases where preclinical results have shown that IL-17A is present at the site and can reproduce in vitro the disease features. This list includes autoimmune diseases such as myositis, lupus and scleroderma. ${ }^{26}{ }^{44}$ The list also includes diseases 
where neutrophils are key contributors to pathogenesis such as neutrophilic skin diseases.

An important issue that will be critical for long-term use is a better understanding of patient heterogeneity, to define patients with an IL-17-driven disease. Indeed, it is easier to expect a positive response to IL-17A inhibition in those patients. Detection of circulating bioactive IL-17A has shown that not all patients with RA have detectable levels at a given time. ${ }^{45}$ Such heterogeneity in IL-17A contribution to disease may explain in part the heterogeneous response to IL-17A inhibition in patients with RA. ${ }^{43}$ Since IL-17A has a priming effect on the response to TNF, acting early may be the way to select to reduce the risk of induction of chronic disease with time. ${ }^{46}$

\section{CONCLUSION}

Drug development is complex and takes time. It took 20 years between the discovery of IL-17A in 1995 and the registration of the first IL-17A inhibitor in 2015! This story of IL-17 discovery to IL-17 targeting is a nice example of translational medicine. Some of the clinical results are as anticipated, while others are not as simple. New pathways and molecules have been discovered during these years. More is to come soon and later.

Funding Part of the work is supported by the IHU prometteur OPERA. PM is a senior member of, and supported by, the Institut Universitaire de France.

Competing interests PM holds a patent on the determination of bioactive IL-17.

Provenance and peer review Commissioned; externally peer reviewed.

Data sharing statement No additional data are available.

Open Access This is an Open Access article distributed in accordance with the Creative Commons Attribution Non Commercial (CC BY-NC 4.0) license, which permits others to distribute, remix, adapt, build upon this work noncommercially, and license their derivative works on different terms, provided the original work is properly cited and the use is non-commercial. See: http:// creativecommons.org/licenses/by-nc/4.0/

\section{REFERENCES}

1. Miossec $\mathrm{P}$, Kolls JK. Targeting IL-17 and TH17 cells in chronic inflammation. Nat Rev Drug Discov 2012;11:763-76.

2. Fossiez $\mathrm{F}$, Djossou $\mathrm{O}$, Chomarat $\mathrm{P}$, et al. T cell interleukin-17 induces stromal cells to produce proinflammatory and hematopoietic cytokines. J Exp Med 1996;183:2593-603.

3. Miossec P, Korn T, Kuchroo VK. Interleukin-17 and type 17 helper T cells. N Engl J Med 2009;361:888-98.

4. Rouvier E, Luciani MF, Mattei MG, et al. CTLA-8, cloned from an activated T cell, bearing AU-rich messenger RNA instability sequences, and homologous to a herpesvirus saimiri gene. $J$ Immunol 1993;150:5445-56.

5. Firestein GS, Zvaifler NJ. How important are T cells in chronic rheumatoid synovitis? II. T cell-independent mechanisms from beginning to end. Arthritis Rheum 2002;46:298-308.

6. Chabaud M, Durand JM, Buchs N, et al. Human interleukin-17: a T cell-derived proinflammatory cytokine produced by the rheumatoid synovium. Arthritis Rheum 1999;42:963-70.

7. Aggarwal S, Gurney AL. IL-17: prototype member of an emerging cytokine family. J Leukoc Biol 2002;71:1-8.

8. Zrioual S, Ecochard R, Tournadre A, et al. Genome-wide comparison between IL-17A- and IL-17F-induced effects in human rheumatoid arthritis synoviocytes. J Immunol 2009;182:3112-20.

9. Hot A, Miossec P. Effects of interleukin (IL)-17A and IL-17F in human rheumatoid arthritis synoviocytes. Ann Rheum Dis 2011;70:727-32.
10. Kleinschek MA, Owyang AM, Joyce-Shaikh B, et al. IL-25 regulates Th17 function in autoimmune inflammation. $J$ Exp Med 2007;204:161-70.

11. Yao Z, Fanslow WC, Seldin MF, et al. Herpesvirus Saimiri encodes a new cytokine, IL-17, which binds to a novel cytokine receptor. Immunity 1995;3:811-21.

12. Gaffen SL. Structure and signalling in the IL-17 receptor family. Nat Rev Immunol 2009;9:556-67.

13. Zrioual S, Toh ML, Tournadre A, et al. IL-17RA and IL-17RC receptors are essential for IL-17A-induced ELR+ CXC chemokine expression in synoviocytes and are overexpressed in rheumatoid blood. J Immunol 2008;180:655-63.

14. Bettelli E, Korn T, Kuchroo VK. Th17: the third member of the effector T cell trilogy. Curr Opin Immunol 2007;19:652-7.

15. Ivanov II, McKenzie BS, Zhou L, et al. The orphan nuclear receptor RORgammat directs the differentiation program of proinflammatory IL-17+ T helper cells. Cell 2006;126:1121-33.

16. Cua DJ, Sherlock J, Chen Y, et al. Interleukin-23 rather than interleukin-12 is the critical cytokine for autoimmune inflammation of the brain. Nature 2003;421:744-8.

17. Noack M, Miossec P. Th17 and regulatory T cell balance in autoimmune and inflammatory diseases. Autoimmun Rev 2014:13:668-77.

18. McGovern JL, Nguyen DX, Notley CA, et al. Th17 cells are restrained by Treg cells via the inhibition of interleukin- 6 in patients with rheumatoid arthritis responding to anti-tumor necrosis factor antibody therapy. Arthritis Rheum 2012;64:3129-38.

19. Kawashima M, Miossec P. Decreased response to IL-12 and IL-18 of peripheral blood cells in rheumatoid arthritis. Arthritis Res Ther 2004;6:R39-45.

20. Puel A, Cypowyj S, Bustamante J, et al. Chronic mucocutaneous candidiasis in humans with inborn errors of interleukin-17 immunity. Science 2011;332:65-8.

21. Toh ML, Kawashima M, Zrioual S, et al. IL-17 inhibits human Th1 differentiation through IL-12Rbeta2 downregulation. Cytokine 2009;48:226-30.

22. Chabaud M, Page G, Miossec P. Enhancing effect of IL-1, IL-17, and TNF-alpha on macrophage inflammatory protein-3alpha production in rheumatoid arthritis: regulation by soluble receptors and Th2 cytokines. J Immunol 2001;167:6015-20.

23. Noack M, Ndongo-Thiam N, Miossec P. Interaction among activated lymphocytes and mesenchymal cells through podoplanin is critical for a high IL-17 secretion. Arthritis Res Ther 2016;18:148.

24. Hot A, Lenief V, Miossec P. Combination of IL-17 and TNFalpha induces a pro-inflammatory, pro-coagulant and pro-thrombotic phenotype in human endothelial cells. Ann Rheum Dis 2012;71:768-76.

25. Hashmi S, Zeng QT. Role of interleukin-17 and interleukin-17-induced cytokines interleukin- 6 and interleukin- 8 in unstable coronary artery disease. Coron Artery Dis 2006;17:699-706.

26. Chevrel G, Page G, Granet C, et al. Interleukin-17 increases the effects of IL-1 beta on muscle cells: arguments for the role of T cells in the pathogenesis of myositis. J Neuroimmunol 2003;137:125-33.

27. Chabaud $\mathrm{M}$, Lubberts $\mathrm{E}$, Joosten $\mathrm{L}$, et al. IL-17 derived from juxta-articular bone and synovium contributes to joint degradation in rheumatoid arthritis. Arthritis Res 2001;3:168-77.

28. Chabaud M, Miossec $\mathrm{P}$. The combination of tumor necrosis factor alpha blockade with interleukin-1 and interleukin-17 blockade is more effective for controlling synovial inflammation and bone resorption in an ex vivo model. Arthritis Rheum 2001;44:1293-303.

29. Kotake S, Udagawa N, Takahashi N, et al. IL-17 in synovial fluids from patients with rheumatoid arthritis is a potent stimulator of osteoclastogenesis. J Clin Invest 1999;103:1345-52.

30. Lubberts E, Van Den Bersselaar L, Oppers-Walgreen B, et al. IL-17 promotes bone erosion in murine collagen-induced arthritis through loss of the receptor activator of NF-kappaB ligand/osteoprotegerin balance. J Immunol 2003;170:2655-62.

31. Osta B, Lavocat $F$, Eljaafari A, et al. Effects of interleukin-17A on osteogenic differentiation of isolated human mesenchymal stem cells. Front Immunol 2014;5:425.

32. Wein MN, Jones $\mathrm{DC}$, Shim JH, et al. Control of bone resorption in mice by Schnurri-3. Proc Natl Acad Sci USA 2012;109:8173-8.

33. Lavocat $F$, Osta B, Miossec $P$. Increased sensitivity of rheumatoid synoviocytes to Schnurri-3 expression in TNF-alpha and IL-17A induced osteoblastic differentiation. Bone 2016;87:89-96.

34. Beringer A, Noack M, Miossec P. IL-17 in Chronic inflammation: from discovery to targeting. Trends Mol Med 2016;22:230-41.

35. Langley RG, Elewski BE, Lebwohl M, et al. Secukinumab in plaque psoriasis-results of two phase 3 trials. N Engl J Med 2014;371: 326-38. 
36. Gordon KB, Blauvelt A, Papp KA, et al. Phase 3 trials of ixekizumab in moderate-to-severe plaque psoriasis. N Engl J Med 2016;375:345-56.

37. Lebwohl M, Strober B, Menter A, et al. Phase 3 studies comparing brodalumab with ustekinumab in psoriasis. $N$ Engl $J$ Med 2015;373:1318-28

38. Baeten D, Sieper J, Braun J, et al. Secukinumab, an interleukin-17A inhibitor, in ankylosing spondylitis. N Engl J Med 2015;373:2534-48.

39. Mease PJ, van der Heijde D, Ritchlin CT, et al. Ixekizumab, an interleukin-17A specific monoclonal antibody, for the treatment of biologic-naive patients with active psoriatic arthritis: results from the 24-week randomised, double-blind, placebo-controlled and active (adalimumab)-controlled period of the phase III trial SPIRIT-P1. Ann Rheum Dis 2017;76:79-87.

40. Danesh MJ, Kimball AB. Brodalumab and suicidal ideation in the context of a recent economic crisis in the United States. J Am Acad Dermatol 2016;74:190-2.

41. Glatt S, Helmer E, Haier B, et al. First-in-human randomised study of bimekizumab, a humanised monoclonal antibody and selective dual inhibitor of IL-17A and IL-17 F, in mild psoriasis. Br J Clin Pharmacol 2016. In press.

42. Lavocat $F$, Maggi L, Annunziato $F$, et al. T-cell clones from Th1 Th17 or Th1/17 lineages and their signature cytokines have different capacity to activate endothelial cells or synoviocytes. Cytokine 2016;88:241-50.

43. Burmester GR, Durez P, Shestakova G, et al. Association of HLA-DRB1 alleles with clinical responses to the antiinterleukin-17A monoclonal antibody secukinumab in active rheumatoid arthritis. Rheumatology (Oxford) 2016;55: 49-55.

44. Crispin JC, Tsokos GC. Interleukin-17-producing T cells in lupus. Curr Opin Rheumatol 2010;22:499-503.

45. Ndongo-Thiam N, Miossec P. A cell-based bioassay for circulating bioactive IL-17: application to destruction in rheumatoid arthritis. Ann Rheum Dis 2015;74:1629-31.

46. Miossec P. IL-17, now an important target for treatment in arthritis. Joint Bone Spine 2016. In press. 